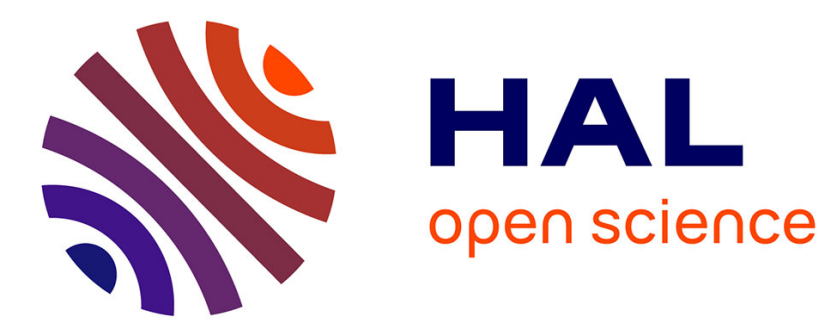

\title{
A microfluidic distribution system for an array of hollow microneedles
}

Antonin Hoël, Nolwenn Baron, Gonzalo Cabodevila, Marie-Caroline Jullien

\section{To cite this version:}

Antonin Hoël, Nolwenn Baron, Gonzalo Cabodevila, Marie-Caroline Jullien. A microfluidic distribution system for an array of hollow microneedles. Journal of Micromechanics and Microengineering, 2008, 18, pp.065019. 10.1088/0960-1317/18/6/065019 . hal-00332019

\section{HAL Id: hal-00332019 https://hal.science/hal-00332019}

Submitted on 20 Oct 2008

HAL is a multi-disciplinary open access archive for the deposit and dissemination of scientific research documents, whether they are published or not. The documents may come from teaching and research institutions in France or abroad, or from public or private research centers.
L'archive ouverte pluridisciplinaire HAL, est destinée au dépôt et à la diffusion de documents scientifiques de niveau recherche, publiés ou non, émanant des établissements d'enseignement et de recherche français ou étrangers, des laboratoires publics ou privés. 


\title{
Microfluidic distribution system
}

\author{
Antonin Hoel $^{1}$, Nolwenn Baron ${ }^{2}$, Gonzalo Cabodevilla ${ }^{2}$ and Marie-Caroline Jullien ${ }^{1}$
}

July 5, 2006

${ }^{1}$ SATIE UMR CNRS 8029, ENS-Cachan, Campus de Ker Lann, 35170 Bruz, France

${ }^{2}$ LPMO UMR CNRS 6174, 32 rue de l'observatoire, 25044 Besançon France

\section{Abstract}

We report a microfluidic device able to control the ejection of fluid through a matrix of out-of-plane microneedles. The device comprises a matrix of open dispensing units connected to needles and filled by a common filling system. A deformable membrane (e.g. in PDMS) is brought into contact with the dispensing units. A pressure exerted on the deformable membrane will close (and thus individualize) each dispensing unit and provoke the ejection of the dispensing unit content through the outlets. A sufficient pressure over the deformable membrane will ensure that all dispensing units will deliver a fixed volume (their content) irrespective of the hydrodynamic pressure outside the dispensing unit outlet. The size of the ensemble matrix of dispensing units, the number of liquid reservoirs, as well as the material can vary depending on the considered application of the device or on the conditions of use. In the present paper, the liquid reservoirs are gemetricaly identical. The geometrical parameters of the device are optimized to avoid as much dead volume as possible, as it was though to handle plasmid DNA solutions which are very expensive. The conception, the fabrication and the experimental results are described in the paper. Our prototype is conceived to inject in a uniform way $10 \mu \mathrm{l}$ of drug through 100 microneedles distributed over one square centimetre.

\section{Introduction}

Since a decade of years, matrix of microneedles have been proposed as an innovative tool to enhance transdermal drug delivery [1]. A lot of work has been done to define the geometrical shape of these needles $[2,3,4]$. However, just a few papers $([5,6,7,8])$ deal with the way to connect this objects to a liquid injection/extraction process. Since these needles are dedicated to inject or extract fluids from the skin, a fluidic system is required to handle the liquids.

The simplest way to inject a liquid through a microneedle matrix is to use a single reservoir that is emptied through the whole matrix. This type of system 
is presented for example in the works of B. Stoeber [5]. While the liquid should mainly pass through the needles with the lower hydrodynamic resistance, such a system should not permit to control the quantity of drug that is injected through each needle. Indeed, apart from the fact that the needles are hardly exactly identical, the skin is not a homogeneous material leading to different hydrodynamic resistance at needle exit, this leads to an inhomogeneous injection of the drug into the skin. Another limit of this simple way to inject liquid through microneedles is pointed out by B. Stoeber : the system does not allow to control precisely the amount of injected drug. Consequently, there are mainly two bottlenecks to face concerning liquids handling : homogeneous spreading through the skin and control of the amount of injected liquid.

In this paper, we report a work in which we describe a microfluidic system that can be connected to a needle matrix, answering to both bottlenecks. This work has been done in the framework of a research project dedicated to the gene treatment of skin diseases by means of microneedles (European project ANGIOSKIN, number LSH-2003-512127). For this kind of treatment, the therapeutical drug has to be equally distributed over the treated skin surface. Furthermore, the DNA solutions used in these treatments are very expensive and are available only in small quantities. In this context, a distribution system that was able to both manipulate small quantities of fluid with minimal losses was mandatory.

Manufacturing microfluidic distribution system is not a technical challenge. For example, an ink jet printer can provide millions of picoliters ink spot in one second. For pharmaceutical use, there are also more specific spotters that are able to manipulate biological fluids such as the VIRTEKs ChipWriterPro ${ }^{T M}$.

However, only few works propose the integration of a fluidic system with a microneedle matrix. Some works present the adaptation of a microfluidic system with a single needle [6] or with a row of five needles [7]. But as far as we know, no work reported the integration of a microfluidic distribution system with a matrix of out-of-plane microneedles.

We report a simple solution to provide a homogeneous injection through a microneedle matrix with the control of the injected quantity of drug. The idea is to adapt a single drug reservoir over each needle, i.e. a single reservoir is split over as many reservoirs as the number of needles. The reservoirs are covered by a membrane that can deflect under pressure and chase the drug through the needle. The dimensions of the reservoir determine the injected volume and the injection is homogeneous while all the reservoirs are pumped out through each single needle.

\section{System}

\subsection{Description}

The system is composed of individual reservoirs disposed on a matrix way. Figure 1 shows a top view of the matrix; the chambers are distributed on one square 


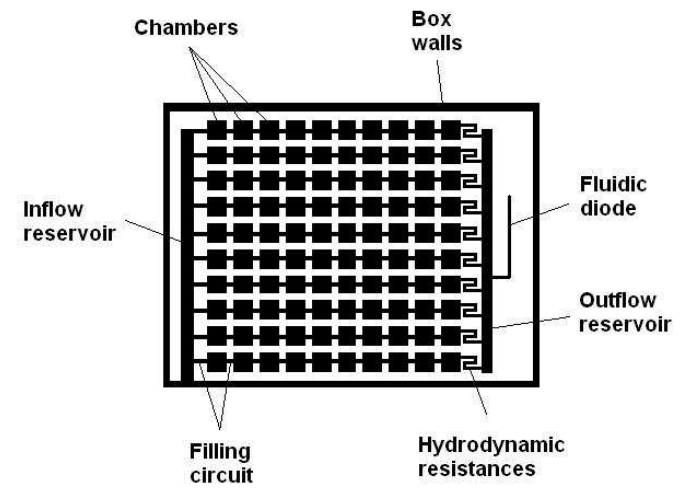

Figure 1: Top view of the system

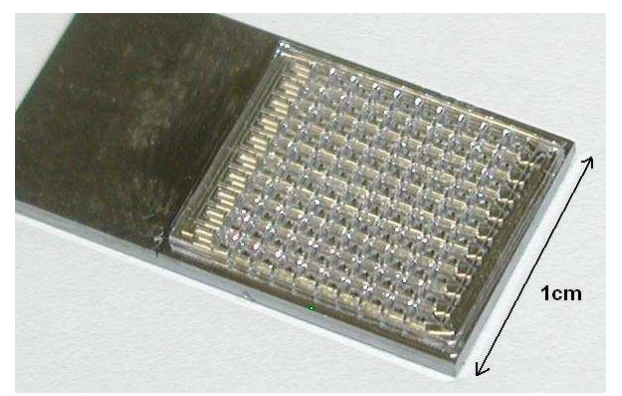

Figure 2: The microfluidic distribution system stuck on an hollowed silicon matrix

centimetre. They are connected to each other via a network of microchannels in order to allow the filling of the whole system from a single sample of product. From the entry to the exit, the filling circuit is composed of five part (Figure 1) : (i) the inflow reservoir, the drug is brought at the entry of each chamber row (this part is connected to the atmospheric pressure), (ii) the chamber matrix connected by small channels (filling circuit), (iii) hydrodynamic resistances which are added in order to obtain a homogenous filling of the system (details are given below), (iv) the outflow reservoir which is only used to connect all the channels to a single exit, and (v) a fluidic diode placed at the connection between the outflow and the pressure reservoir (contained into a box). The diode at the end prevents the drug to flow back through the filling circuit during the injection, i.e., while the pressure is increased into the box in order to deflect the membrane used to chase the liquid reservoir content throught its counterpart needle.

The reservoirs and the filling circuit are covered by a hyperelastic membrane. The mechanical properties of the membrane are chosen to achieve very high deformations in order to pump out a maximum of the chambers content through 
the needles when it is under pressure. Figure 2 is a picture of the microfluidic system stuck on a silicon needle matrix.

Figure 3 is a transversal sketch of the system presenting the way our system is able to both fill the dispensing units from a single entrance as well as eject the content of each dispensing units through the corresponding needles. The details of the principle are given in next section.

\subsection{Principle}

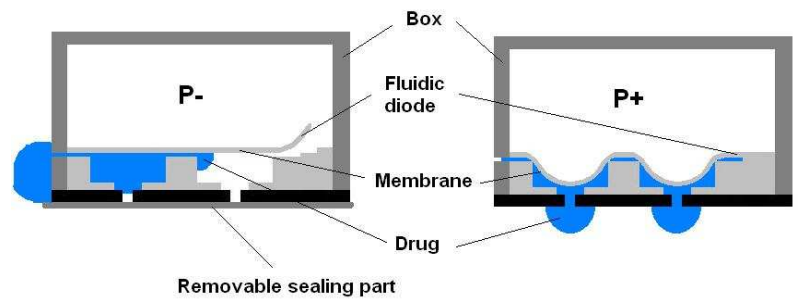

Figure 3: Left : filling of the system, right : ejection of the drug

The system is filled from a single drop of drug. Its volume is the one of the total system volume. The drop is brought at the entry of the filling circuit by hand. In order to prevent any leak from the chambers to the outlet of the needles, the needles are driven into a soft material in order to close them while filling the chambers matrix (Figure 3, left). The drug drop is then sucked into the chamber matrix by means of a reduction of the pressure into the box which is connected to the exit of the filling circuit. During this stage, the fluidic diode is open.

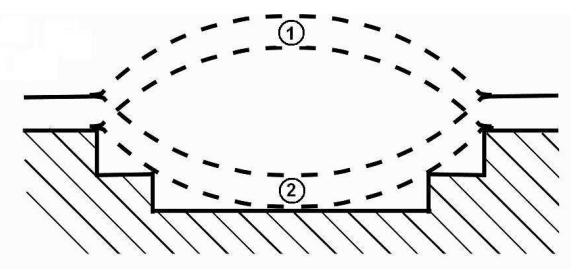

Figure 4: Filling circuit cross section

The filling circuit is located just under the membrane. Figure 4 presents a cross section of it. During filling, the membrane deflects upwards and the hydrodynamic resistance of the filling circuit is very low (case 1 in figure 4). When the pressure inside the box is reversed for the ejection of the fluid, the membrane deflects downwards and the section of the channels is dramatically reduced (case 2 in figure 4 ). This conducts to a very high hydrodynamic resistance in the channel which prevents the drug to use this pathway preferably to the needle one. This geometry assures that the system behaves like a fluidic 
circuit during the filling and like a matrix of individual chambers during the ejection, which is one of the core of the system.

For the ejection, the soft material which closes the needles is removed and the pressure in the box is increased (Figure 3, right). This closes the fluidic diode, makes the chambers individual units, and while the membrane deflects into the chambers it ejects the drug through the needles.

It is a single use system. In one hand, for hygienic reasons, the system cannot be used twice without been sterilized, and in the other hand, after a first use, some drug remains into the system and prevents a good filling of it. It is however possible to rinse it with water and let it dry up before a new use.

\subsection{Fabrication}

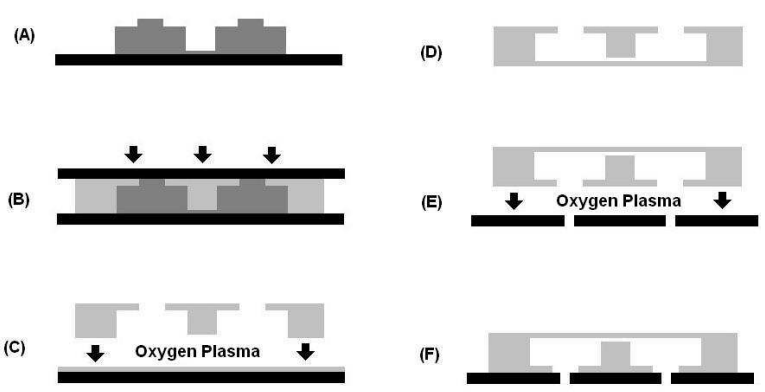

Figure 5: (A) Mould realization by SU8 photolithography, (B) Backing into a press system, (C) Gluing on the membrane using an oxygen plasma, (D) Removal and cutting of the system, (E) Gluing on the microneedle matrix using an oxygen plasma, (F) Packaging

To manufacture the system, we first made a mould for both the chamber matrix and the filling circuit. The soft photolithography technology with a thick photoresist (SU8) was used. In a first step the wafer is coated with a $100 \mu \mathrm{m}$ photoresist layer and is further insulated to make the filling circuit; then a second layer of $200 \mu \mathrm{m}$ or more is coated over the first layer to make the chambers. To obtain a photoresist layer as thick as $200 \mu \mathrm{m}$, SU82100 is spincoated and baked several times. Each spincoating adds a layer about 100 $\mu$ m thick.

The mould is then developed and CHF3 is deposited on it by a plasma treatment. This treatment makes the removal of PDMS easier after casting. More than two steps of photoresist coating and insulation can be processed in order to achieve a pyramidal structure. A pyramidal structure assures a better sealing of the filling circuit (figure 4) and reduces the dead volume (i.e. liquid that is lost) into the chambers after injection. Usually, the filling circuit is realised in two steps and the chambers in three. 
Figure 5 describes the different manufacturing steps. First, the commercially PDMS prepolymer and catalyser (RTV 615, Bayer silicones) are mixed and deposited on the mould. The PDMS is then degased under vacuum to eliminate bubbles created during preparation. To make the chambers pierce the PDMS sheet, the mould is put into a pressing system during backing. In parallel, the membrane is made by speencoating PDMS on a clean wafer. At a speed of $2200 \mathrm{tr} / \mathrm{min}$ during thirty seconds, we can realize a thirty micrometer thick membrane. The membrane is then glued on the chambers after being activated by oxygen plasma.

The fluidic diode is made at this stage. A square of thin paper is put on the system at the place of the diode to prevent the gluing of the membrane. The trap is then cut off with a cutter and the paper is removed.

Lastly, the PDMS system is glued on the microneedles matrix using again an oxygen plasma activation and the whole system is packed into a box that can be connected to a pressure system. During assembling, care is done in order to leave the fluid inlet outside the box, while all the microfluidic system is contained into the box (filling circuit, matrix chamber, fluid outlet and fluidic diode).

\section{Study}

\subsection{Material}

The material used for the system is PDMS. This polymer is easily mouldable at the microscale and has hyperelastic properties. The mechanical properties of this material are very variable. Specifically the shear modulus, which determines the propensity of the membrane to deflect, can vary from $100 \mathrm{kPa}$ to $3 \mathrm{MPa}$ [9]. These mechanical properties depend on several factors such as the proportion of mixed prepolymer and catalyser, the time and temperature into the oven or the brand of the PDMS that is used.

To allow the membrane to have the maximal deformation, we need a low shear modulus. This modulus was measured using traction assays. For a mixture of 15 volumes prepolymer for 1 volume catalyser, a shear modulus of 250 $\mathrm{kPa}$ (baking $2 \mathrm{~h}$ at $70 \mathrm{C}$, brand RTV) was measured. For a 10:1 mixture with the same other parameters, the shear modulus was about $400 \mathrm{kPa}$. With this in mind, we realized the membrane of the system with a 15:1 mixture and the chambers with a 10:1 mixture. Different catalyser ratio helps the gluing of the membrane on the chambers.

The PDMS is naturaly a hydrophobic material. However some treatments such as the oxygen plasma activation make it temporaly hydrophilic. It is therefore possible to choose the surface properties of the PDMS (hydrophilic vs hydrophobic). 


\section{$4.2 \quad$ Filling system}

The filling circuit is designed to fill entirely all the chambers of the matrix. There are two main difficulties with this part : bubbles may stay inside the chambers and some chambers may not be filled at all due to small local inhomogeneity of the material.

To prevent the formation of bubbles inside the chambers during the filling, the walls have to be hydrophobic. With hydrophilic walls, the filling circuit is instantaneously filled by capillarity without any pressure. But the capillarity does not fill the whole chambers, and these bubbles cannot be chased out of the system. With hydrophobic walls, the drug firstly fills completely the chambers before going further into the filling circuit in which the ratio surface/bulk is higher.

While the walls are hydrophobic, when a row of chambers is completely filled until the exit of the filling circuit, the drug will stop to fill the remaining empty chambers. The entire drug will pass through the way already achieved. For this reason, all the chambers must be filled before the drug reaches the exit. However, practically, all the rows of chambers are not filled at the same speed. This is probably due to small local inhomogeneities in the materials properties. To assure the complete filling of the matrix, hydrodynamic resistances are added at the end of the filling circuit. Since the liquid has more affinity to fill lower surface/ratio parts, the drug starts to fill the hydrodynamic resistances when all the chambers are filled.

\subsection{System sizing}

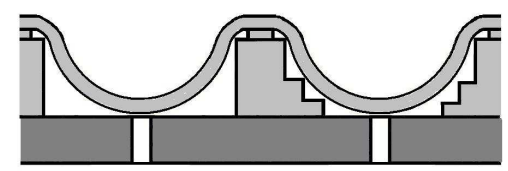

Figure 6: Cross section of the chambers during injection. Left without a pyramidal structure and right the pyramidal structure reduces the dead volume

Sizing of the system is built on several criteria. First of all, the size of the chambers is linked to the quantity of drug that has to be injected and the topology has to prevent from loss of drug. However, losses are unavoidable and the topology has to permit a good filling of the chambers. A finite element model was done to evaluate the influence of the geometry on the injected volume and to the losses into the system.

The model was built using the COMSOL Multiphysics software. It consists of a single chamber covered by a membrane. As the PDMS is a hyperelastic polymer, the model cannot use standard solid mechanics model that does not include large deformations. Therefore, the Neo Hookean model was chosen. This model is an extension of Hooke's law for the case of large deformations; it 
is useable for plastics and rubber-like substances. The model of neo-Hookean solid assumes that the extra stresses due to deformation are proportional to Finger tensor (1)

$$
\mathbf{T}=-p \mathbf{I}+G \mathbf{B}
$$

where $\mathbf{T}$ is the stress tensor, $\mathrm{p}$ the pressure, $\mathbf{I}$ the unity tensor, $\mathrm{G}$ a constant equal to shear modulus, and $\mathbf{B}$ the Finger tensor. The strain energy for this model is (2):

$$
W=\frac{1}{2} G I_{B}
$$

where $\mathrm{W}$ is potential energy and $I_{B}=\operatorname{tr}(\mathbf{B})$ is the trace (or first invariant) of Finger tensor B. The Neo-Hookean model only needs the shear modulus to characterize the material since PDMS is supposed to be incompressible.

These computations were used as a tool to determine the influence of chamber geometry on losses that have to be minimized and also to get an order of magnitude of the required pressure to apply in order to obtain the expected membrane displacement, which was found to be typically about one bar. At a given pressure, there are two parameters that were considered to evaluate the quality of the geometry: the displacement of the membrane and the ratio of the remaining volume of the chamber divided by the volume occupied by the deflected membrane (further noted Vi). This parameter has to be minimized since it evaluates losses.

Cylindrical and squared chambers shape have been tested, and the ratio was the same. As soon as a square shape leads to a better compaction, all experiments were performed with this geometry.

As expected, increasing the shear modulus leads to a lower membrane deflection while the other parameters are set. Thus, these numerical simulations give us orders of magnitude to set for the experiments in order to obtain the required deflection. Therefore, in order to have the smallest $\mathrm{Vi}$, we used the lowest shear modulus $(250 \mathrm{kPa})$ to evaluate the geometry efficiency. In the same way, a thicker membrane has a lower deflection, and thicker chambers are more difficult to empty. Therefor, after fixing a minimal thickness for the chamber lateral walls $(200 \mu \mathrm{m}$, due to the fabrication limits) all the surface of the system is occuped by the chambers that are as thin and wide as possible. For the prototype, in wich the needles are $1 \mathrm{~mm}$ spaced, the chambers have a square shape of $800 \mu \mathrm{m}$ in side. The thicker these chambers are, the more product the system can inject, but the dead volume goes higher to.

Furthermore, thanks to the model, we could obtain the evaluation of the membrane deformation shape while it is under pressure. With these data, we can adjust the height of steps, to manufacture a pyramidal like shape, done into the chamber to reduce the dead volume (figure 6).

\subsection{Results}

On figure 7 the microfluidic system has been stuck on a hollowed microneedle matrix. Figure 7.a shows the microneedle matrix before ejection, while the 

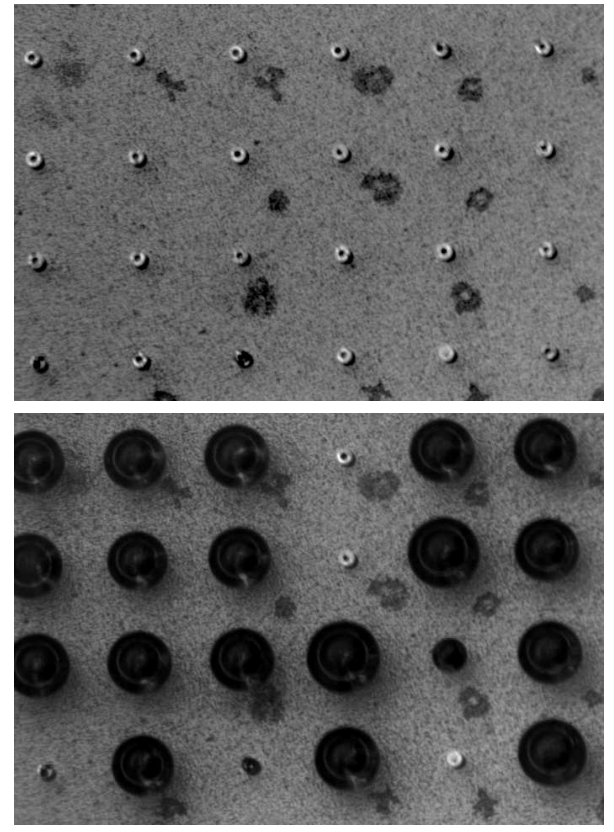

Figure 7: Above : microneedles before the ejection, and bottom : ejection of ink through the needles

microfluidic system is already filled with a died liquid (ink). On Figure 7.b, ejection has been processed and the figure shows that all steps have been successfull at $80 \%$ : chambers filling and homogeneous ejection for the most importants. This result shows that the microfluidic system successfully fulfils the following requests: homogeneous injection of a liquid and control of the ejected volume of each drop.

On figures 7 , it is also noticeable that ink did not go through some of the needles $(20 \%)$. These needles are probably clogged, and that may be caused by several reasons: the needles may have not been completely pierced during the fabrication or impurity may have obstructed the needles during the fabrication process. There is also a possibility that the chambers above these needles were not correctly filled; however, the good filling of the other chambers tend to refute this hypothesis since a chamber which is not filled cannot fill next chambers since they are connected via the filling circuit.

We can however verify that the ink passes through most of the needles and that the ejected ink volume is almost the same for each needle (120 nl \pm 10 $\mathrm{nl}$ ). The control of the quantity and the homogeneity of ejection is therefore achieved. Ejection test into the skin were not achieved until now while the needles we used did not pierce the skin.

The main default of the system is that many and long fabrication stages are required for a single use. It is therefore difficult to make a lot of assays with the 
system.

\section{Conclusion}

A microfluidic distribution system has been designed, fabricated and tested. We successfully perform the manipulation of small liquid volumes (inital drop of $20 \mu \mathrm{l}$ ) and further inject the liquid through a microneedle matrix with a minimal loss of the drug (about $50 \%$ of the used drug). It provides a homogenous injection over the entire matrix and allows controlling the quantity of drug that is injected $(120 \mathrm{nl} \pm 10 \mathrm{nl}$ for each needle, i.e. a total injected volume about 10 $\mu \mathrm{l})$.

More precisely, the system has been concieved to handle DNA solutions. Now, making DNA pass through a microchannel is a method to obtain a random fragmentation of it [10]. Therefore electrophoresis tests were done to verify the integrity of DNA molecules after passing through the system. The asseys showed that a $7 \mathrm{kbp}$ plasmide DNA did not fractionate in our conditions of use.

However, the manufacture of the whole system is a bit tricky. In particular, the making of the diode require a manual operation witch is difficult at this scale. Solutions remain to be found in order to make the realization of the system easier and more repetitive.

\section{Aknowledgement}

This work was done within the framework of the European project ANGIOSKIN (project number: LSH-2003-512127). We wish to thank Lluis Mir (coordinator of the project) to make us participating in this highly stimulating project as well as for fruitfull discussions.

\section{References}

[1] Mark R. Prausnitz. Microneedles for transdermal drug delivery. Advanced Drug Delivery Reviews, 56:581-587, 2004.

[2] E.V. Mukerjee, S.D. Collins, R.R. Isseroff, and R.L. Smith. Microneedle array for transdermal biological fluid extraction and in situ analysis. Sensor and Actuators A, 114:267-275, 2004.

[3] Achim Trautmann, Patrick Ruther, and Oliver Paul. Micronnelde arrays fabricated using suspended etch mask technology combined with fluidic through wafer vias. In MEMS 2003, Kyoto, Japan, January 2003.

[4] P. Zhang and G.A. Jullien. Microneedle arrays for drug delivery and fluid extraction. In Proceedings of the 2005 International conference on MEMS, Nano and Smart Systems (ICMENS '05), 2005.

[5] Boris Stoeber and Dorian Liepmann. Design, fabrication and testing of a mems syringe. In Solid State Sensor and Actuator Workshop, June 2002. 
[6] Jefrey D. Zahn, Ajay A. Deshmukh, Alexandros P. Papavasiliou, Albert P. Pisano, and Dorian Liepman. An integrated microfluidic device for the continuous sampling and analysis of biological fluids. In Proceedings of 2001 ASME International Mechanical Engineering, November 2001.

[7] Seung-Joon Paik, Jung-Min Lim, Ilwoo Jung, Yonghwa Park, Sangwon Byun, Seok Chung, Kukjin Chun, Junkeun Chang, and Dongil Dan Cho. A novel microneedle array integrated with a pdms biochip for microfluid systems. In Transducers '03, June 2003.

[8] Shyh-Chyi Kuo and Yukon Chou. A novel polymer microneedle arrays and pdms micromolding technique. Tamkang Journal of Science and Engineering, 7(2):95-98, 2004.

[9] J.C. Lötters, W. Olthuis, P.H. Veltink, and P. Bergveld. The mechanical properties of the rubber elastic polymer polydimethylsiloxane for sensor applications. Journal of Micromechanics and Microengineering, 7:145-147, 1997.

[10] Yvonne R. Thorstenson, Scott P Hunicke-Smith, Peter J. Oefner, and Ronald W. Davis. An automated hydrodynamic process for controlled, unbiased dna shearing. Genome Research, 8:848-855, 1998. 